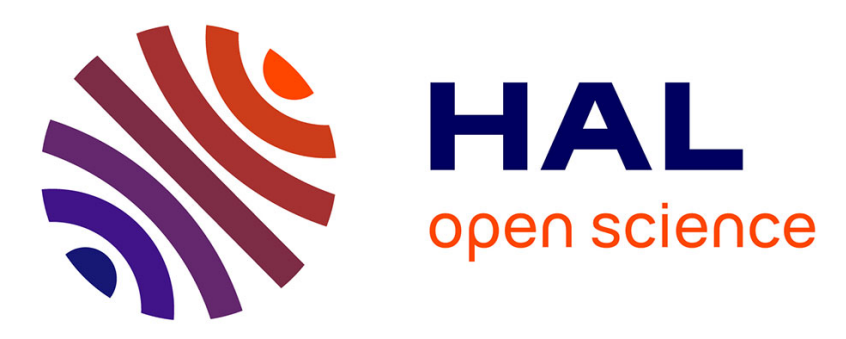

\title{
Study of Blind Rendez-vous in Low Power Wireless Sensor Networks
}

Affoua Thérèse Aby, Alexandre Guitton, Michel Misson

\section{To cite this version:}

Affoua Thérèse Aby, Alexandre Guitton, Michel Misson. Study of Blind Rendez-vous in Low Power Wireless Sensor Networks. VTC Spring (IEEE Vehicular Technology Conference), 2014, Seoul, South Korea. hal-01789140

\author{
HAL Id: hal-01789140 \\ https://hal.science/hal-01789140
}

Submitted on 9 May 2018

HAL is a multi-disciplinary open access archive for the deposit and dissemination of scientific research documents, whether they are published or not. The documents may come from teaching and research institutions in France or abroad, or from public or private research centers.
L'archive ouverte pluridisciplinaire HAL, est destinée au dépôt et à la diffusion de documents scientifiques de niveau recherche, publiés ou non, émanant des établissements d'enseignement et de recherche français ou étrangers, des laboratoires publics ou privés. 


\title{
Study of Blind Rendez-vous in Low Power Wireless Sensor Networks
}

\author{
Affoua Thérèse Aby ${ }^{(1,2)}$, Alexandre Guitton ${ }^{(3,2)}$, Michel Misson ${ }^{(1,2)}$ \\ (1) Clermont Université, Université d'Auvergne, LIMOS, BP 10448, F-63000 Clermont-Ferrand, France \\ (2) CNRS, UMR 6158, LIMOS, F-63175 Aubière, France \\ (3) Clermont Université, Université Blaise Pascal, LIMOS, BP 10448, F-63000 Clermont-Ferrand, France \\ Emails: \{aby,guitton,misson\}@ sancy.univ-bpclermont.fr
}

\begin{abstract}
In wireless sensor networks (WSNs) applications such as environmental monitoring, it is essential to design protocols that are energy efficient and that are scalable. The main technique to reduce energy consumption is to use a duty-cycle, where nodes periodically go to sleep. However, the implementation of such a duty-cycle often requires nodes to be synchronized, which is difficult to achieve in a scalable manner. In this paper, we study the delay of blind rendez-vous for unsynchronized nodes, that is the delay required for two unsynchronized nodes to be active at the same time. There are several major advantages of blind rendez-vous: nodes do not need to share a prior knowledge, nodes do not need to be synchronized, and the support for network dynamicity (due to node mobility or failure) is simplified. We show through simulations that the delay to achieve a blind rendez-vous is reasonable in many scenarios.
\end{abstract}

\section{INTRODUCTION}

Wireless sensor networks (WSNs) are a promising solution for environmental monitoring due to their easy deployment, energy autonomy and low cost. Examples of such applications include the monitoring of volcanoes [1], of bird nests [2], of fields [3], and of bridges [4]. These types of applications require the deployment of a large number of energy-efficient sensor nodes, and the auto-configuration of these nodes. Several MAC protocols have been proposed in this area to achieve a trade-off between energy consumption and delay.

The IEEE 802.15.4 [5] standard has been proposed for low-power, low-rate wireless personal area networks, which includes WSNs. The standard operates in two modes: the beacon-enabled mode and the non beacon-enabled mode. In the beacon-enabled mode, all nodes are synchronized: they wake up to communicate, and go back to sleep simultaneously to save energy. The main drawback of this mode is that the synchronization mechanism is costly in terms of energy and is not scalable. In the non beacon-enabled mode, nodes wake up when they have a packet of data to transmit and go into sleep mode after transmission. The main drawback of this mode is that it requires a large number of nodes to remain active to receive packets, which causes a significant energy consumption. Thus, IEEE 802.15.4 is not able to achieve a good trade-off between energy consumption and scalability, due to the overhead and complexity of the synchronization.

In this paper, we study the behaviour of IEEE 802.15.4 in beacon-enabled mode, when the requirement of synchronized beacons is removed. Each node is active according to its own random schedule. Blind rendez-vous occur when two nodes in the same vicinity are active at the same time, which occurs randomly. There are several major advantages to blind rendezvous: nodes do not need to share a prior knowledge of their schedule, they do not need to be synchronized, and the support for network dynamicity (due to node mobility or node failure) is simplified. During these rendez-vous, it can also be noted that contention for the medium is reduced, as some nodes are still sleeping. These advantages come at the cost of long delays before blind rendez-vous, which is the focus of this study.

The remainder of this paper is organized as follows. Section II describes the state of the art of related MAC protocols. Section III describes how to remove the requirement of synchronized beacons from IEEE 802.15.4 in beacon-enabled mode. Section IV presents our simulations results. Finally, Section V summarizes our work.

\section{STAte OF ThE ART}

Most energy-efficient MAC protocols are based on a sequence of active and inactive periods, called duty-cycle. Nodes remain active for a given duration and go back to sleep for the rest of the time, which saves energy. These MAC protocols can be divided into synchronized and unsynchronized.

\section{A. Synchronized MAC protocols}

The IEEE 802.15.4 protocol [5] in beacon-enabled mode is one of the most common low-power MAC protocols for WSNs. Each full-function device (FFD) sends a beacon with a period called $B I$ (for beacon interval). When a reducedfunction device (RFD) receives a beacon, it starts its activity period for a duration called $S D$ (for superframe duration). The ratio $S D / B I$ defines the duty-cycle of the protocol. Note that all RFDs are in range of a FFD, and are synchronized by the beacon. All nodes share the same activity period. The medium is accessed using slotted CSMA/CA (carrier-sense multiple access with collision avoidance).

In D-MAC [6], nodes are synchronized according to their depth on a collection tree. When nodes of depth $d$ are in a transmission slot, nodes of depth $d-1$ are in a reception slot. The synchronization is also performed using the tree topology. In S-MAC [7] and [8], each node propagates periodically its time schedule to its neighbors. Thus, nodes can determine 
when to be active or inactive, depending on whether they have to communicate with a given neighbor or not.

In Z-MAC [9], a TDMA (time-division multiple access) approach is used. A slot is assigned to all nodes in the configuration phase, and is used in case of high contention. In G-MAC [10], time is divided into three periods: a collection period (where the medium is accessed by CSMA/CA), a period of traffic indication (whose role is to maintain synchronization between nodes) and a distribution period (where the medium is accessed by TDMA). All nodes have simultaneous activities during the first and the second periods, and non-simultaneous activities during the third period. In SEA-MAC [11], the authors propose a synchronous duty-cycle MAC protocol which allows nodes to adjust their duty-cycle according to the dynamic traffic load.

\section{B. Unsynchronized MAC protocols}

The IEEE 802.15.4 protocol in non beacon-enabled mode allows nodes to communicate without requiring a synchronization mechanism. When an RFD has to send data to an FFD, it simply wakes up and transmits the data. This requires, however, the FFDs to be active all the time, which consumes energy. The medium is accessed using the nonslotted CSMA/CA algorithm, a slightly modified version of slotted CSMA/CA. Although the non beacon-enabled mode allows nodes to have non-simultaneous activities, it cannot be used in practice due to its large energy requirements.

In B-MAC [12], nodes are not synchronized but wake up periodically for a short duration. When a sender node has to communicate to a receiver node, the sender node sends a long preamble before its frame (which makes B-MAC a senderinitiated protocol). When the receiver wakes up, it detects the preamble and stays active until the end of the preamble and the reception of the frame. While this approach yields good performance, nodes have to stay awake frequently in order to receive frames, and therefore the energy consumption of $\mathrm{B}-\mathrm{MAC}$ relies heavily on the traffic.

In X-MAC [13] and [14], the same approach as B-MAC is used. Instead of using a long preamble, each sender sends several small frames, which allows the receiver to go back to sleep as soon as it has received the frame, rather than having to wait for the preamble. RI-MAC [15], ABD [16], PW-MAC [17], EM-MAC [18] and WiseMAC [19] focus on a receiver-initiated approach. In RI-MAC, the receiver initiates the communication by using a low power probing, where the receiver sends a beacon to express its ability to receive data packets. RI-MAC reduces channel occupation (as it does not require nodes to send preambles), but generates high energy consumption. The ABD protocol provides a broadcast service in RI-MAC protocol. PW-MAC reduces the listening time of the sender in RI-MAC, by having each node compute its awakening times according to a pseudo-random number generator rather than according to a fixed schedule. The drawback of PW-MAC is that sending the beacons before packet transmission generates overhead, and introduces a delay when listening the channel. In EM-MAC [18], a node computes its awakening schedule using a pseudo-random generator. A node independently decides its wake-up time and exchange channel. The wake-up channel is not necessarily the same as the channel for exchanging data. In EM-MAC, the sender also knows the parameters of the pseudo-random generator and receiver wake-up channel. EM-MAC inherits the shortcomings of PW-MAC, and more, in EM-MAC, each node invokes twice a pseudo-random generator, hence, generating an additional overhead. In WiseMAC, preambles are used, but their lengths is reduced by allowing the sender to wake up before the beginning of the activity period of the receiver.

\section{Blind rendez-vous}

Blind rendez-vous refer to the ability of a node to be active at the same time as another node, and to detect it without prior knowledge. In [20], the authors show that only few MAC protocols operate using blind rendez-vous. The authors study an algorithm where rendez-vous is detected by having a node scan the channel to detect the activity of the other node. Once a rendez-vous occurs, the node that has detected it stays on the same channel. In this paper, we have different assumptions: the rendez-vous has to be detected each time it occurs, and we generate explicit messages instead of detecting the activity of nodes (which is supposed to be limited).

\section{IMPLEMENTATION OF BLIND RENDEZ-VOUS}

In this section, we describe how IEEE 802.15.4 in beaconenabled mode can be changed to work without requiring a beacon synchronization. In this new version, each node decides of its own schedule randomly and independently, at each cycle.

\section{A. Description of the modification of IEEE 802.15.4}

In the MAC layer of the beacon-enabled mode of IEEE 802.15.4 (in its original version), nodes are synchronized by the beacons. The activities of nodes are simultaneous, as shown on Fig. 1 for a duty-cycle of $25 \%$. We denote this approach $A_{s p}$, where the nodes are synchronized and their activities are periodically repeated at the same time. In this approach, nodes meet systematically in each cycle. However, this approach causes high contention because multiple nodes attempt to access the channel at the same time, and requires synchronization.

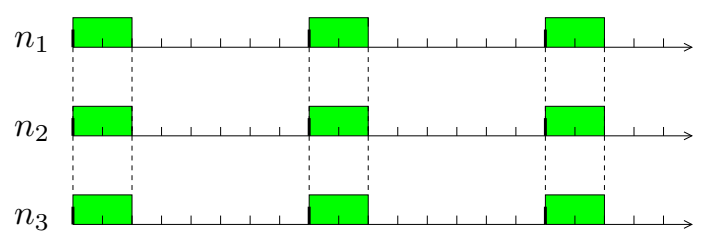

Figure 1. Activity of three nodes (in time) when the duty-cycle is $25 \%$, and all activities are synchronized and periodic.

If the synchronization procedure of the beacon-enabled mode of IEEE 802.15.4 is removed, it is possible to consider an approach where activities are periodic, but unsynchronized. We denote this approach $A_{u p}$. The start time of the activity of each node is independent, as shown in Fig. 2. This approach 
has the advantage to reduce the contention for the channel, because few nodes are active at the same time, and also does not require synchronization. However, the major drawback of $A_{u p}$ is the high probability that some pairs of nodes never communicate directly (see pairs $\left(n_{1}, n_{3}\right)$ and $\left(n_{2}, n_{3}\right)$ on Fig. 2).

Note that in Fig. 2, the time slots for each node are still synchronized. In practice, this is not required. Each time two nodes share an activity period, one time slot is generally lost at the beginning of the common activity period, and another at the end of the common activity period, due to synchronization issues. However, as these time slots are expected to be very short (they can have the same length as a backoff period, which is $320 \mu \mathrm{s}$, time base of the IEEE 802.15.4 standard) with respect to the much longer common activity duration, this phenomenon can be neglected.

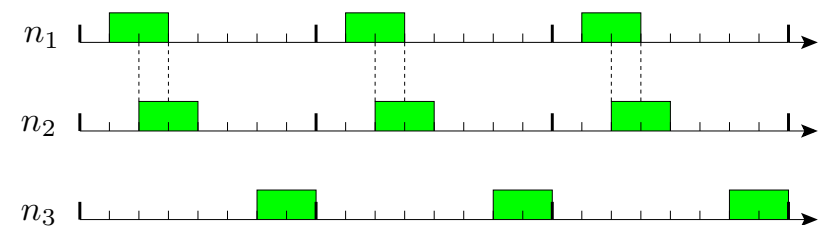

Figure 2. Activity of three nodes (in time) when the duty-cycle is $25 \%$, when nodes start their activities independently but in a periodic manner.

The mechanism we study shifts randomly the starting time of the activity of each node, at each cycle. We denote this approach $A_{u a}$, as activities are unsynchronized and also aperiodic. $A_{u a}$ is shown on Fig. 3. This approach has many advantages: any pair of nodes will eventually be able to have a rendez-vous, contention is low (as the average number of nodes active at the same time is low), and no synchronization is required. Note that broadcasting is also possible, as the probability that all nodes are active simultaneously is not null (even if it is low). However, the fact that few nodes are generally active simultaneously reduces the contention for the medium and the probability of collisions, which in turn reduces the probability to miss a detection. The main drawback of our approach is that it can yield long delays before a given pair of nodes share a common activity period.

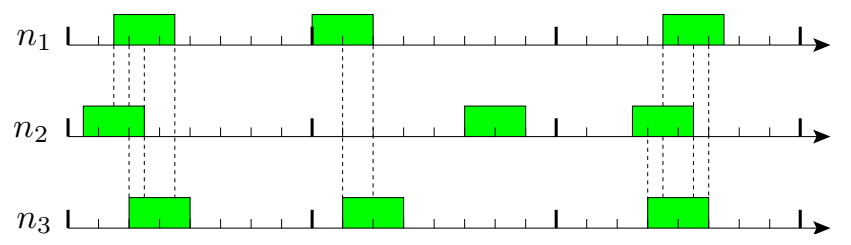

Figure 3. Activity of three nodes (in time) when the duty-cycle is $25 \%$, when nodes start their activities independently and randomly in each cycle.

\section{B. Technical details on the implementation}

The mechanism can be implemented easily from IEEE 802.15.4 as follows. Each node knows its duty-cycle $e$ and the cycle duration $b$. Each node schedules its activities according to its own schedule, and each activity period lasts for a duration $s=e . b$. The start of the activity duration is randomly chosen in the interval $[0 ; b-s[$. When the activity duration starts, the node wakes up, sends a beacon message, and waits to detect neighbors before sending data messages, as shown on Fig. 4. Neighbors are detected (and thus, rendezvous are generated) when the node receives a beacon message from another node. Beacon and data messages are sent using unslotted CSMA/CA.

To detect a rendez-vous, a minimum duration (called $t$ in the following) is required. Let us consider two nodes, $n_{1}$ and $n_{2}$, and let us assume that $n_{1}$ is active before $n_{2}$ (for a given cycle). For $n_{1}$ to detect the rendez-vous with $n_{2}, n_{1}$ has to wait for the beginning of the activity of $n_{2}$, and then for the reception of the beacon of $n_{2}$ (which is delayed as beacons are sent using unslotted CSMA/CA). Thus, time $t$ includes the average time required to send one packet (i.e., the beacon) in unslotted CSMA/CA (assuming that the contention is relatively low). A study conducted by Niek et al. [21] showed that this time varies between $2 \mathrm{~ms}$ and $6 \mathrm{~ms}$, in the case of a perfect channel. To take into consideration lossy channels and beacon retransmissions, we set the value of $t$ to $15.36 \mathrm{~ms}$. This value corresponds to the minimum duration of a superframe in IEEE 802.15.4, and is expected to be sufficient to exchange a few frames among nodes, even if several nodes are contending for the medium. The impact of $t$ on the network performance is left for future work. We assume that as soon as two nodes can share a common activity period of length at least $t$, one of them can detect the rendez-vous. An analytical evaluation of the delay has been proposed in [22], in a slightly different case where each node initially chooses a random value for its cycle duration, computes its duty-cycle accordingly, and activates its radiomodule periodically afterwards. The main differences with this paper are the following: in this paper, we assume that all nodes have the same cycle duration $b$ and activate their radio-module randomly within each cycle duration, and our delay evaluation is more accurate as it takes into account the contention delay (and not only the delay before a common activity).

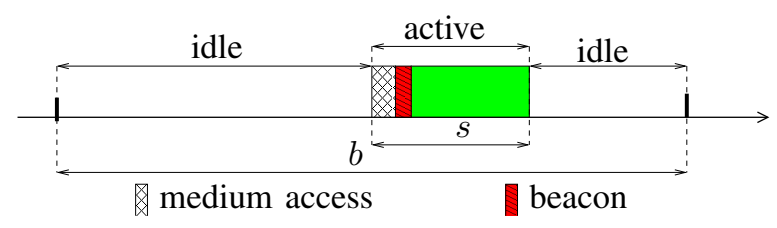

Figure 4. Zoom on the activity of a node.

\section{Reduction of the delay to detect a rendez-vous}

The delay required to detect a rendez-vous is the main focus of this study, as it is the main issue of the mechanism. To reduce this delay, the activity can be fragmented: instead of having one activity of duration $s=e . b$ randomly located every $b$ time units (where $e$ is the duty-cycle), nodes can have several activities, each of duration $e . b / f$, randomly located every $b / f$ time units. Note that the duty-cycle in the second case is $(e . b / f) /(b / f)=e$, as it is in the first case. Figure 5 
shows an example with three nodes, with $b=8, e=25 \%$ and $f=2$. Note that each activity lasts for $e . b / f=1$ time unit, and is randomly located once every $b / f=4$ time units.

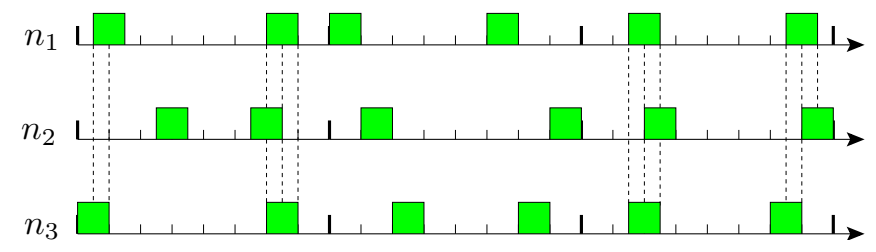

Figure 5. Activity fragmentation into $f=2$, with a duty-cycle of $25 \%$.

\section{Performance EVAluation}

We conducted simulations in order to evaluate the delay before blind rendez-vous, for several scenarios. The simulation settings are the following. Backoff slots of $320 \mu$ s are used, as in the IEEE 802.15.4 standard. For the sake of simplicity, backoff slots are synchronized: to deal with unsynchronized backoffs, an additional delay of $320 \mu$ s should be added to all results (but this value is negligeable with respects to the total delay we obtain). Time for each node is expressed using a large array $a: a_{n}[i]$ is equal to 1 if node $n$ is active at time-slot $i$, and $a_{n}[i]$ is equal to 0 otherwise. Nodes $n_{1}$ and $n_{2}$ share a common activity at time-slot $i$ if $a_{n_{1}}[i]=a_{n_{2}}[i]=1$. To account for lossy channel, we assume that the rendez-vous can be detected if the nodes share an activity of $t=15.36 \mathrm{~ms}$, that is 48 backoff slots. Results are averaged over 300 repetitions, and each repetition is a sequence of cycles that lasts for 1 hour. We vary the duration of the cycle $b$, as well as $e$.

In all the simulations, the evaluated delay is the time before a common activity of two nodes. This delay does not take into account the medium access delay required for a node to transmit a beacon, nor the probability that the beacon can be missed by an active receiver due to the channel. We expect the medium access delay to be lower than $t=15.36 \mathrm{~ms}$, as our mechanism yields a low contention, and thus we consider that the medium access delay can be neglected. We also consider that the two nodes are in range of each other, and that channel losses due to the propagation conditions are low. Losses due to collisions should also be limited, as the probability that many nodes are active at the same time is low.

Figure 6 shows the average delay for two nodes to detect a rendez-vous for at least $t=15.36 \mathrm{~ms}$, as a function of the duration of the activity cycle $b$, for our protocol. Three dutycycles are shown: $5 \%, 15 \%$ and $25 \%$. As expected, the delay increases when $b$ increases: when the cycles are long, it takes more time for nodes to share a common activity. Also, the delay increases when $s$ decreases: when the duty-cycle is low, common activity occurs less often, which in turn increases the delay. For a cycle of $60 \mathrm{~s}$ and a duty-cycle of $25 \%$, nodes wait on average less than $60 \mathrm{~s}$. For a cycle of $10 \mathrm{~s}$ and a duty-cycle of $5 \%$, nodes wait on average about $80 \mathrm{~s}$. When the duty-cycle is $15 \%$ or more, our approach achieves small delays: it takes less than two global cycles for two nodes to achieve a rendez-vous.

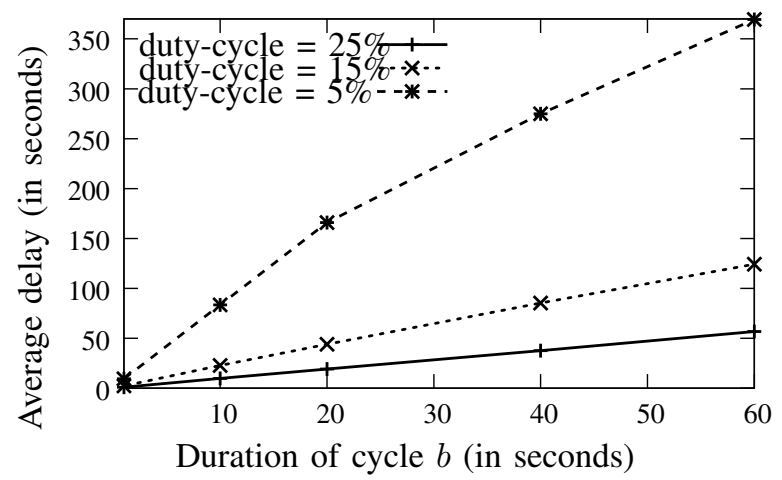

Figure 6. Average delay for two nodes to detect a rendez-vous.

Figure 7 shows the impact of the fragmentation of the activity on the average delay, as a function of the duration of the cycle $b$, for a duty-cycle of $5 \%$. For a cycle of $b=60 \mathrm{~s}$, the average delay is more than $350 \mathrm{~s}$ when there is no fragmentation $(f=1)$, and is only $120 \mathrm{~s}$ when the activity is fragmented four times $(f=4)$. With the fragmentation of the activity, the delay has been significantly reduced. This result shows that without changing the duty cycle of nodes, it is possible to reduce the delay for blind rendez-vous.

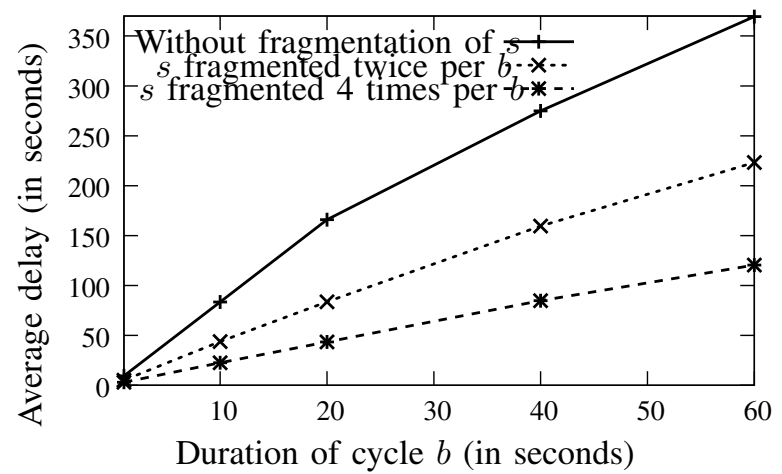

Figure 7. Impact of activity fragmentation when the duty-cycle is $5 \%$.

Figure 8 shows the impact of the fragmentation of the activity on the average delay, as a function of the duration of the cycle $b$, for a duty-cycle of $25 \%$ (rather than $5 \%$ for Figure 7). Again, the reduction of the delay is very important as $f$ increases. For a cycle of $b=60 \mathrm{~s}$, the delay can be reduced from about $57 \mathrm{~s}$ to $14 \mathrm{~s}$, which is about a quarter of the whole activity cycle. Even if a delay of $14 \mathrm{~s}$ seems to be large, it is important to remember that nodes are active only $25 \%$ of the time, and that nodes experience this delay only when they have packets to send, which is expected to be rare.

It is not possible to fragment the activity too many times. Indeed, the more the activity is fragmented, the shortest duration nodes have in common. When $f$ becomes too large, the common duration becomes too small, and nodes cannot detect rendez-vous anymore. We choose $f$ so that it achieves a good tradeoff between the delay (which reduces when $f$ increases) and the common activity duration (which decreases when $f$ increases, as shown on Figure 9). We propose to 


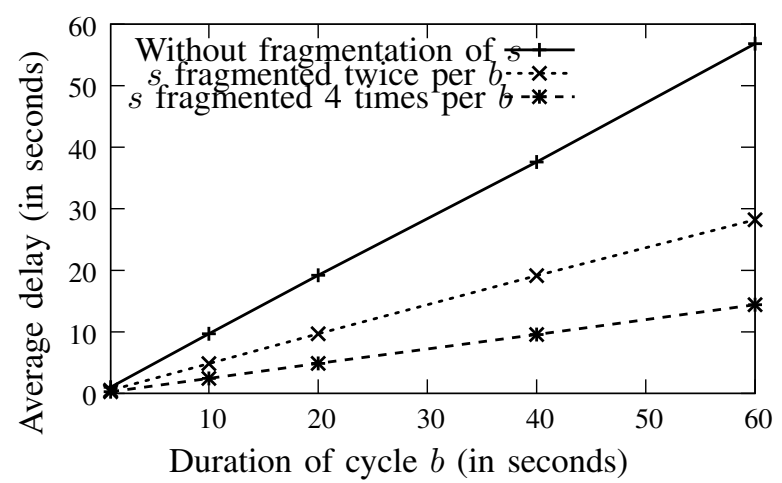

Figure 8. Impact of activity fragmentation when the duty-cycle is $25 \%$.

choose the largest value of $f$ for which the common activity duration exceeds $t=15.36 \mathrm{~ms}$. The computation of the exact value of $f$ is left for future work.

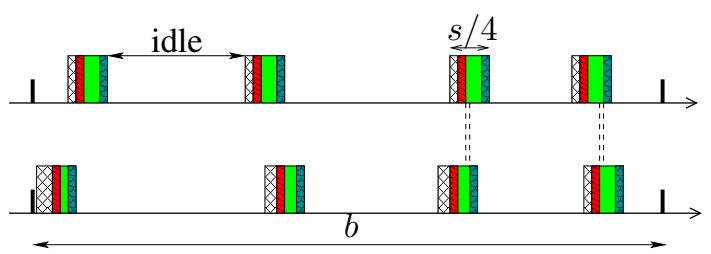

圈medium access beacon $t<$ data+ack times

Figure 9. Node activity when $s$ is fragmented four times per $b(e=25 \%)$

\section{CONCLUSION}

The IEEE 802.15.4 protocol in beacon-enabled mode is a good candidate for WSNs, as it aims at reducing the energy consumption of nodes. However, the synchronization required for IEEE 802.15.4 is costly and difficult, and raises energy consumption and scalability issues. Thus, we propose to remove this requirement by allowing nodes to have their activities start at a random time within each global cycle. We explain how nodes can achieve blind rendez-vous, and we compute how long nodes have to wait (on average) before these rendez-vous. Even when the duty-cycle is low (such as $5 \%$ ), the duration before rendez-vous can be kept small (in this case, the average is always at most twice the duration of a cycle). The advantages of blind rendez-vous are that contention is reduced (as in general, only few nodes are active simultaneously), and network dynamicity can be supported easily. Our future work consists in determining the optimal cycle duration (i.e., the optimal value of the fragmentation of the cycle) that ensures that rendez-vous can be detected, in realistic conditions (such as with lossy links and congestion).

\section{ACKNOWLEDGMENT}

This is Laboratory of Excellence ClerVolc contribution number 89.

\section{REFERENCES}

[1] W. Geoffrey, J. Jeff, R. Mario, L. Jonathan, and W. Matt, "Monitoring volcanic eruptions with a wireless sensor network," in European Workshop on Wireless Sensor Networks (EWSN'05), 2005.

[2] S. Robert, M. Alan, P. Joseph, A. John, and C. David, "An analysis of a large scale habitat monitoring application," in ACM Conference on Embedded Networked Sensor Systems (SenSys), 2004, pp. 214-226.

[3] J. Hart and K. Martinez, "Environmental sensor networks: A revolution in the Earth system science?" Earth Science Reviews, vol. 78, no. 3, pp. 177-191, 2006.

[4] T. Nagayamaa, M. Ushitab, and Y. Fujinoa, "Suspension bridge vibration measurement using multihop wireless sensor networks," in East Asia-Pacific Conference on Structural Engineering and Construction (EASEC). Elsevier, 2011, pp. 761-768.

[5] IEEE 802.15, "Part 15.4: Wireless medium access control (MAC) and physical layer (PHY) specifications for low-rate wireless personal area networks (WPANs)," ANSI/IEEE, Standard 802.15.4 R2006, 2006.

[6] G. Lu, B. Krishnamachari, and C. Raghavendra, "An adaptive energyefficient and low-latency MAC for data gathering in sensor networks," in Ad Hoc and Sensor Networks, April 2004.

[7] W. Ye, J. Heidemann, and D. Estrin, "An energy-efficient MAC protocol for wireless sensor networks," in IEEE Infocom, 2002, pp. 1567-1576.

[8] S. Ganeriwal, I. Tsigkogiannis, H. Shim, V. Tsiatsis, B. Srivastava M., and D. Ganesan, "Estimating clock uncertainty for efficient duty-cycling in sensor networks." IEEE/ACM Transactions on Networking, 2009.

[9] I. Rhee, A. Warrier, M. Aia, and J. Min, "A hybrid MAC for wireless sensor networks," in ACM SenSys, San Diego, California, USA, November 2005 .

[10] M. Brownfield, Y. Gupta, and N. Davis, "Wireless sensor network denial of sleep attack," in IEEE Workshop on Information Assurance, June 2005.

[11] Y. Z. Zhao, C. Y. Miao, and M. Ma, "An energy-efficient self-adaptive duty cycle MAC protocol for traffic-dynamic wireless sensor networks," in Wireless Personal Communications, 2012, p. 1287-1315.

[12] J. Polastre, J. Hill, and D. Culler, "Versatile low power media access for wireless sensor networks," in ACM Sensys, November 2004.

[13] M. Buettner, Y. Gary, V., E. Anderson, and R. Han, "X-MAC: A short preamble MAC protocol for duty-cycled wireless sensor networks," in ACM Sensys, Boulder, Colorado, USA, November 2006.

[14] P. Tom, H. Gertjan, B. Maarten, and L. Koen, "The MAC framework: redefining mac protocols for wireless sensor networks," in Wireless Networks, February 2010, p. 2013-2029.

[15] Y. Sun, O. Gurewitz, and B. Johnson, D., "RI-MAC: a receiver-initiated asynchronous duty cycle MAC protocol for dynamic traffic loads in wireless sensor networks," in Proc. ACM SenSys, 2008.

[16] S. Yanjun, G. Omer, D. Shu, T. Lei, and J. David, B., "ADB: an efficient multihop broadcast protocol based on asynchronous dutycycling in wireless sensor networks," in In Sensys'09: Proceedings of the 7 th International Conference on Embedded Networked Sensor Systems, 2009, pp. 43-56.

[17] Y. Sun, O. Gurewitz, and D. Johnson, "PW-MAC: An energy-efficient predictive-wakeup MAC protocol for wireless sensor networks," in Proc. INFOCOM, April 2011, pp. 1305-1313.

[18] T. Lei, S. Yanjun, G. Omer, D. Shu, and J. David, B., "EM-MAC: A dynamic multichannel energy-efficient MAC protocol for wireless sensor networks," in MobiHoc, Paris, France, May 2011, pp. 1-11.

[19] A. El-Hoiydil, J.-D. Decotigniel, and J. Hernandez, "WiseMAC: An ultra low power MAC protocol for multi-hop wireless sensor networks," in Algorithmic Aspects of Wireless Sensor Networks, ser. LNCS, vol. 3121. Springer Berlin / Heidelberg, 2004, pp. 18-31.

[20] J. Misic, V. B. Misic, M. S. I. Khan, and M. M. Rahman, "Properties of blind rendezvous in channel hopping cognitive piconets," in IEEE VTC-Fall, 2013.

[21] D. Niek, V., L. Benoît, M. Pieter, D., M. Ingrid, D. Bart, and D. Piet, "Throughput and delay analysis of unslotted IEEE 802.15.4," Journal of Networks, vol. 1, pp. 20-28, 2006.

[22] A. T. Aby, A. Guitton, and M. Misson, "MAC mechanism for a scalable wireless sensor network using independent duty cycles," in New and smart Information Communication Science and Technology to support Sustainable Development, 2013. 